\title{
छs \\ Disentangling Effects of Nuclear Structure in Heavy Element Formation
}

\author{
D. J. Hinde, R. G. Thomas, ${ }^{*}$ R. du Rietz, A. Diaz-Torres, M. Dasgupta, M. L. Brown, M. Evers, \\ L. R. Gasques, ${ }^{\dagger}$ R. Rafiei, and M. D. Rodriguez \\ Department of Nuclear Physics, Research School of Physical Sciences and Engineering, The Australian National University, \\ Canberra, ACT 0200, Australia
}

(Received 21 March 2008; published 20 May 2008)

\begin{abstract}
Forming the same heavy compound nucleus with different isotopes of the projectile and target elements allows nuclear structure effects in the entrance channel (resulting in static deformation) and in the dinuclear system to be disentangled. Using three isotopes of Ti and $\mathrm{W}$, forming ${ }^{232} \mathrm{Cm}$, with measurement spanning the capture barrier energies, alignment of the heavy prolate deformed nucleus is shown to be the main reason for the broadening of the mass distribution of the quasifission fragments as the beam energy is reduced. The complex, consistently evolving mass-angle correlations that are observed carry more information than the integrated mass or angular distributions, and should severely test models of quasifission.
\end{abstract}

The formation of new, superheavy elements [1] requires the fusion of two massive nuclei. After contact of the two colliding nuclei, the dinuclear system is pictured as moving in deformation space over a potential energy surface (PES). The system may reach compact (mononuclear) shapes inside the fission barrier, and thus have the possibility of forming a heavy element in its ground state. Alternatively, before reaching a compact shape, the system may reseparate into two heavy fragments, intermediate in mass between the projectile and target nuclei. This is called quasifission [2,3]. The probability of fusion (forming a new nucleus with a compact near-equilibrium shape) is determined by many variables. Those believed to play a major role include the mass-asymmetry of the two colliding nuclei, the charge of the heavy element being formed, and the neutron and proton shell structure encountered during the fusion process [4]. The detailed role and significance of shell structure is the most complex and open question.

Shell effects after contact can be categorized as dynamic or static. Nonequilibrium dynamic effects, associated with the diabatic motion of the nucleons at avoided crossings of their molecular orbitals, may effectively result in timedependent shell structures [5] that can influence the early collective motion of the dinuclear system. After thermal equilibration, the well-known static shell structures affect the PES, and also the inertia and the strength of energy dissipation (and thus the magnitude of shape fluctuations), which together determine the motion over the PES. In spontaneous and low energy fission of heavy nuclei, the role of (static) shell structures is well known. Fission mass yields and total kinetic energy distributions, and the existence and properties of fission isomers, can be understood as resulting from local structure in the PES caused by energetically favorable shell structures in the nascent fragments, both for spherical and deformed shapes [6]. It is expected (and observed) that as the excitation energy $E_{x}$ increases, these shell effects are exponentially attenuated.
This strong dependence on $E_{x}$ may be a key to isolating their role in quasifission dynamics. However, there are uncertainties regarding the effective energy scale of the attenuation [7], which is critical to predict the influence of shell structure on quasifission, where $E_{x}$ is often $>20 \mathrm{MeV}$.

In collisions of heavy nuclei, a further complication often arises, resulting from nuclear structure effects in the entrance channel. The heavy reaction partner may have a large static deformation. This is caused by unfavored shell effects for a spherical shape, compared with a gain in energy (shell stability) for a deformed shape. This deformation affects the entry point into the PES. For nuclear collisions involving prolate nuclei, when the deformation axis is aligned with the projectile nucleus, the dinucleus is very elongated at contact, whereas it is more compact if antialigned. In experiments, by choice of the bombarding energy, only the aligned orientation (low beam energy) or all orientations (higher energy) can be selected [8-10]. Measurements have consistently shown that the lower energies are associated with the largest deviation of fission properties from the expectations for fusion-fission [10-14], as well as showing suppression of the yield of heavy elements following capture $[15,16]$. Both observables should be associated with an increased probability of quasifission $[10,11]$.

Having two different effects of nuclear shell structure (in the entrance channel and in the dinuclear system), both of which are expected to be most significant at the lowest bombarding energies, leads to a confusing situation, as the same measured effects may be interpreted within completely different frameworks. This is a significant problem in understanding the formation of superheavy elements, as all target nuclei heavier than $\mathrm{Pb} / \mathrm{Bi}$ have large prolate deformations. It is reactions with these nuclei that have given evidence of formation of the heaviest elements, with atomic number up to 118 [1]. However, at the same time, in 
studies of a wide range of such reactions forming heavy nuclei, measured mass and kinetic energy distributions have often been interpreted within a framework where shell effects in the dinucleus are taken to play the dominant role [17].

An experimental method is needed to allow the effects of shell structure in the entrance channel to be separated from the effects of shell structure of the dinuclear system. A new experimental approach is presented, using different isotopes of the projectile and target elements to form the same compound nucleus $(\mathrm{CN})$, which allows these individual effects to be disentangled.

To carry out the measurements, pulsed beams $(\simeq 1.5 \mathrm{~ns}$ FWHM) of ${ }^{46,48,50} \mathrm{Ti}$ in the energy range $222-241 \mathrm{MeV}$, provided by the ANU 14UD electrostatic accelerator, bombarded enriched targets of ${ }^{186} 184182 \mathrm{~W}$ respectively, all reactions forming the composite nucleus ${ }^{232} \mathrm{Cm}$. These W isotopes all have large prolate deformations $\left(\beta_{2} \approx 0.24\right)$. The targets were $\sim 50 \mu \mathrm{g} / \mathrm{cm}^{2}$ in thickness, evaporated onto $15 \mu \mathrm{g} / \mathrm{cm}^{2} \mathrm{C}$ backings (facing downstream), and angled at $30^{\circ}$ to the beam axis. Reference measurements were made, forming the neighboring $\mathrm{CN}^{234} \mathrm{Cm}$, using ${ }^{32} \mathrm{~S}$ beams of 159-192 MeV, bombarding an enriched target of ${ }^{202} \mathrm{Hg}$ of $\sim 20 \mu \mathrm{g} / \mathrm{cm}^{2}$. Fission fragments were measured in coincidence, in two $28 \mathrm{~cm} \times 36 \mathrm{~cm}$ position sensitive multi-wire proportional counters [11], located on opposite sides of the beam axis. The timing foils were at closest $18 \mathrm{~cm}$ from the target, giving a wide angular coverage of $5^{\circ}-80^{\circ}$ and $50^{\circ}-125^{\circ}$. This permitted complete coverage of all mass-splits between projectile and target, for a range of center-of-mass angles $\left(\theta_{\text {c.m. }}\right)$ between $40^{\circ}$ and $140^{\circ}$. The azimuthal acceptance of the back angle (trigger) counter was close to $90^{\circ}$ for all angles; thus, the number of events observed at any $\theta_{\text {c.m. }}$ is proportional to $d \sigma / d \theta_{\text {c.m. }}$.

The benefits of forming the same $\mathrm{CN}$ with different isotopes of the projectile and target elements are (i) the Coulomb interaction is identical for each reaction, eliminating one variable in the dynamics and (ii) the fusion $Q$ values for the reactions are different. For the ${ }^{50} \mathrm{Ti}+{ }^{182} \mathrm{~W}$ and ${ }^{46} \mathrm{Ti}+{ }^{186} \mathrm{~W}$ reactions, the $Q$ values differ by $13 \mathrm{MeV}$, with the ${ }^{48} \mathrm{Ti}+{ }^{184} \mathrm{~W}$ reaction lying between. Beam energies were carefully chosen, corresponding to approximately $E / V_{B}=1.01,1.05$, and 1.09 of the respective capture barrier energies (Bass barriers [18] with a radius shift of $0.3 \mathrm{fm}$ ) for each reaction. Together with the different $Q$ values, it was then possible to form the $\mathrm{CN}$ at the same $E / V_{B}$ with different excitation energies $E_{x}$, as well as at different $E / V_{B}$ for the same $E_{x}$, allowing the effects of $E / V_{B}$ and $E_{x}$ to be isolated. Although the different neutron to proton ratios $(N / Z)$ for the projectile and target nuclei in each reaction could slightly affect the fusion and quasifission dynamics, the effect should be small, as the difference in the number of nucleons is small, and $N / Z$ undergoes rapid equilibration, as known from studies of deepinelastic reactions.
The mass-angle distributions (MAD) were extracted by first transforming the position and time information from the two MWPCs to allow determination of the velocity vector of each coincident particle. The time origin for each beam energy was determined from the requirement that the average center-of-mass velocity of the fission events should match that expected for full momentum transfer in the capture process. The required velocity correlations for a binary reaction [11] allowed rejection both of random coincidences and reactions with the target backing, giving clean spectra for the desired reaction. The mass ratio $M_{R}=$ $M_{\text {Back }} /\left(M_{\text {Back }}+M_{\text {Front }}\right)$ was determined from the ratio of the velocities in the center-of-mass frame [11]. Since both fragments were detected for each event, the mass-angle matrix was populated both at $\left(M_{R}, \theta_{\text {c.m. }}\right)$ and at $\left(1-M_{R}\right.$, $\left.\pi-\theta_{\text {c.m. }}\right)[13,14]$. The measured MAD for the Ti-induced reactions are shown in Fig. 1, together with one distribution for the ${ }^{32} \mathrm{~S}+{ }^{202} \mathrm{Hg}$ reaction for comparison.

The distributions show (i) the fission $M_{R}$ distributions for all the Ti reactions are much wider than for ${ }^{32} \mathrm{~S}+$ ${ }^{202} \mathrm{Hg}$, (ii) the Ti reactions show a complex correlation of $M_{R}$ with $\theta_{\text {c.m. }}$, depending strongly on $E / V_{B}$, but weakly on the projectile (and thus $E_{x}$ ), and (iii) the strong correlation of mass with angle shows that the reaction time is typically less than the rotation time $\left(\sim 10^{-20} \mathrm{~s}\right)$, in agreement with previous conclusions regarding these Ti reactions $[19,20]$. The MAD show a marked similarity to those measured for the reactions of $\mathrm{S}, \mathrm{Cl}$ and $\mathrm{Ca}$ with ${ }^{238} \mathrm{U}$ [21] (taking into

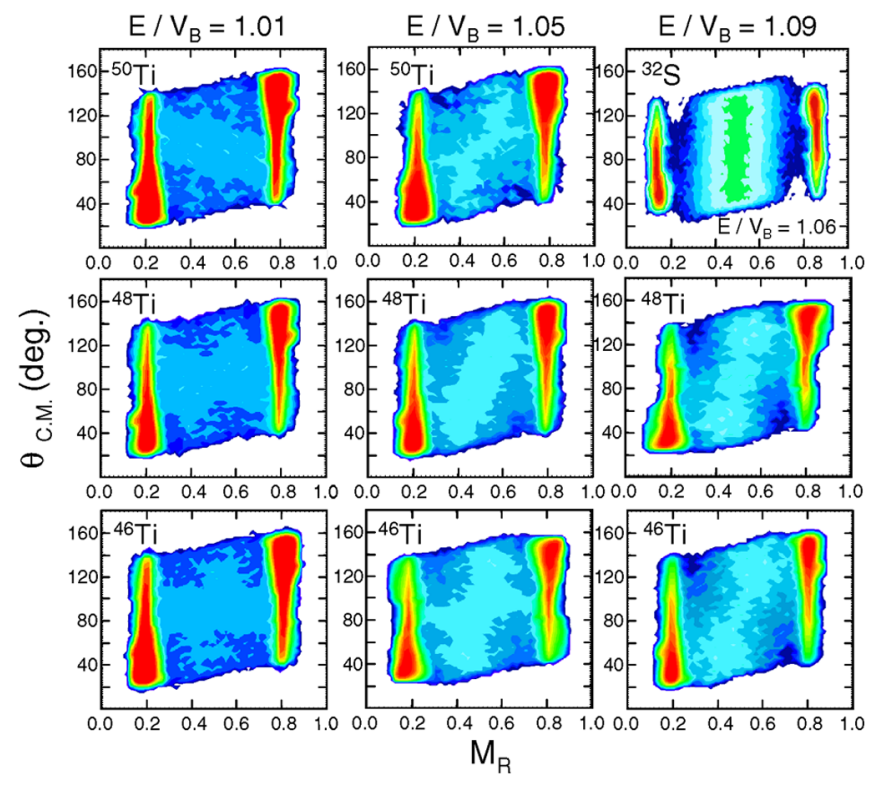

FIG. 1 (color online). Contour plots of counts as a function of mass ratio $M_{R}$ and center-of-mass angle $\theta_{\text {c.m. }}$. Columns correspond to the same $E / V_{B}$, while rows correspond to the same projectile-target combination, except for the top right panel, which shows data for the ${ }^{32} \mathrm{~S}+{ }^{202} \mathrm{Hg}$ reference reaction. Between the intense scattering peaks, the fissionlike events for the Ti-induced reactions exhibit a complex mass-angle correlation, which evolves consistently with $E / V_{B}$. 
account the inverse kinematics used in that work), suggesting that the complex structure may be universal, reflecting common behavior in the dynamics of reactions involving heavy deformed nuclei. As first demonstrated in Refs. [3,21], MAD with a wide angular range give unique insights into the evolution of the dinuclear system in shape and time. Their prediction should be a high priority for future developments of realistic quasifission transport models.

To make model-independent comparisons of the results of the current measurements, $M_{R}$ distributions (Fig. 2) have been generated from each MAD, for an angle cut $40^{\circ}<\theta_{\text {c.m. }}<140^{\circ}$, where full detector efficiency prevails. Because of the double population of the MAD for each event, and the symmetry of the angle cut around $\theta_{\text {c.m. }}=90^{\circ}$, the $M_{R}$ distributions are symmetric about $M_{R}=0.5$; cuts asymmetric about $\theta_{\text {c.m. }}=90^{\circ}$ would give asymmetric distributions. There are suggestions in the distributions of detailed structure, but to characterize these distributions by a single number, they have each been fitted with a Gaussian function. The standard deviations $\sigma_{M}$ of the best-fitting Gaussians, with statistical uncertainties, are shown in Fig. 3 for all the $\mathrm{Ti}$ and $\mathrm{S}$-induced measurements. For the lowest $E_{x}$, a flat distribution $\left(\sigma_{M}=\right.$ $\infty)$ is acceptable.

Figure 3(a) shows the results plotted as a function of the $\mathrm{CN}$ excitation energy $E_{x}$. Each Ti isotope gives a significantly different $\sigma_{M}$ for the same $E_{x}$. If the increase in mass-width as the beam energy decreases resulted exclusively from the increasing influence of shell structure on the PES, all the Ti reactions should lie on a single curve. The data show that this cannot be the case. To investigate

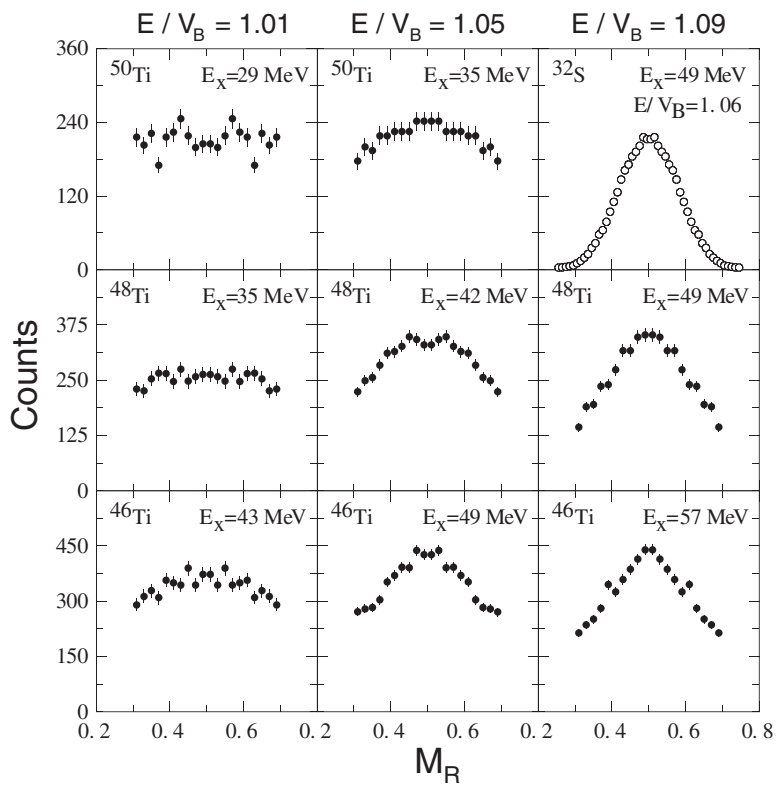

FIG. 2. Projected mass-ratio distributions for each of the distributions shown in Fig. 1 , for $40^{\circ}<\theta_{\text {c.m. }}<140^{\circ}$. $E_{x}$ indicates the excitation energy of the $\mathrm{CN}$. the alternative correlation, between $\sigma_{M}$ and deformation alignment, the $\sigma_{M}$ are shown as a function of $E / V_{B}$ in Fig. 3(b). The correlation between the different Ti isotopes is much stronger, indicating that deformation alignment is the major reason for the increase in the quasifission masswidth as the beam energy falls (also seen qualitatively in Figs. 1 and 2). This conclusion was also made recently in Refs. $[13,22]$, where reactions of ${ }^{48} \mathrm{Ca}$ and ${ }^{48} \mathrm{Ti}$ with ${ }^{154} \mathrm{Sm}$ (deformed) were compared with reactions where deformation plays no role.

To investigate this theoretically, adiabatic potential energies in the mass-asymmetry degree of freedom have been calculated at the contact distance [23], using the finite range liquid drop model (FRLDM), with shell and pairing corrections for these molecular shapes determined by applying Strutinski's method to two-center shell model calculations [24]. The effect of static deformations has been included by using calculated [25] ground state quadrupole deformations. Because of a restriction to axially symmetric molecular shapes, for the aligned (elongated) contact configurations, prolate fragments have been taken, whereas to estimate the energies for the antialigned (compact) configurations, oblate fragments have been taken. These potentials are shown in Fig. 4, by the full and dotted curves, respectively, where the calculation reference energy is the FRLDM energy of the spherical CN; the FRLDM energies for spherical fragments are shown by the thin line.

In the aligned configuration, the potential energy drives each reaction towards mass-symmetry, which should rap-
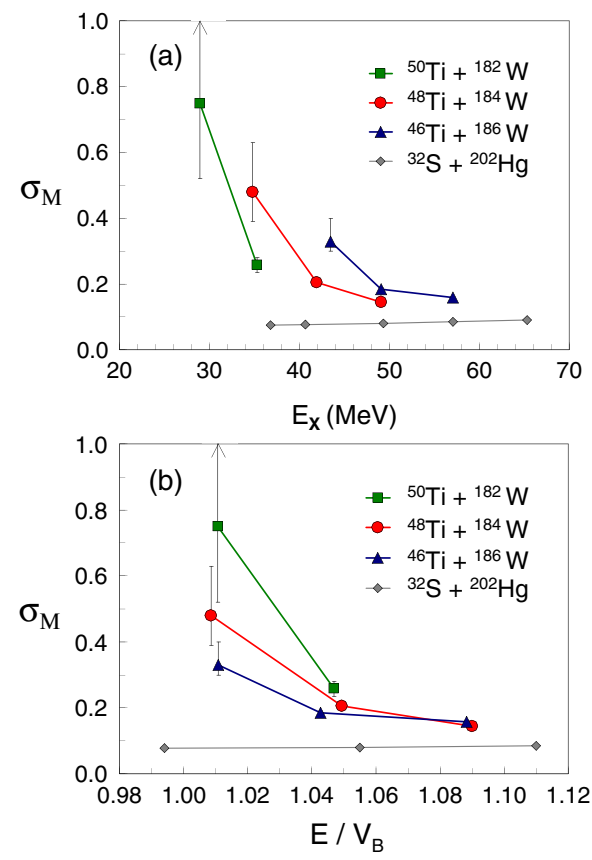

FIG. 3 (color online). Widths of the Gaussian function bestfitting the measured mass-ratio distributions shown in Fig. 2. The dependence on excitation energy of the $\mathrm{CN}$ is shown in (a), while the dependence on the ratio of the beam energy to the average barrier energy $\left(E / V_{B}\right)$ is shown in (b). 


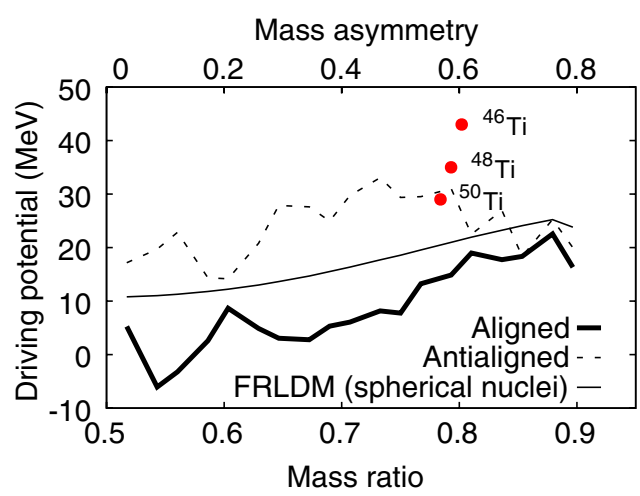

FIG. 4 (color online). Driving potentials at the contact configuration as a function of the mass ratio, calculated with the two-center shell model for deformation aligned and antialigned configurations (see text). Entry points for the lowest energies (aligned configuration) are labeled by projectile. The mass asymmetry $\eta=2 M_{R}-1$.

idly lead to quasifission, as the elongation is far outside the fission saddle point. For the antialigned configuration, the potential is more favorable to initially increasing the mass ratio (absorbing the projectile), leading to more compact configurations, allowing more time for mass equilibration. This should result in narrower mass distributions of the final binary fragmentation. The calculations support the interpretation from experiment, and agree with general arguments [11], as well as recent calculations [26], for a shift in $M_{R}$ of the Businaro-Gallone point depending on alignment.

The measured mass-widths at the lower $E / V_{B}$ [Figs. 2 and 3(b)] show a systematic decrease from ${ }^{50} \mathrm{Ti}$ to ${ }^{46} \mathrm{Ti}$. This cannot be associated with Ti deformation, since ${ }^{50} \mathrm{Ti}$ is spherical, but gives the largest $\sigma_{M}$. The change in $M_{R}$ of the PES entry points (Fig. 4) seems qualitatively too small to be the explanation. The effect may, however, be associated with the increase in $E_{x}$ from ${ }^{50} \mathrm{Ti}$ to ${ }^{46} \mathrm{Ti}$ causing damping of shell effects with excitation energy. In the calculated driving potentials (Fig. 4), the structures essentially result from the variation in ground state shapes of the two fragments (and are rather weakly affected by other microscopic corrections); the peak at $M_{R}=0.6$ (aligned configuration) is associated with near-spherical fragments. For low excitation energy, where the level densities reflect most strongly the potential energy surface, this peak may inhibit further mass flow, enhancing the mass yields at $M_{R}>0.6$, and thus contributing to the increasing width of the mass distributions with reducing $E_{x}$. It may also be significant that the measured $M_{R}$ distribution for ${ }^{50} \mathrm{Ti}$ (lowest $E_{x}$ ) shows fluctuations. Further measurements should show whether these are significant and can be related to nuclear structure.

A new experimental approach, using different isotopes of the projectile and target elements (forming the same $\mathrm{CN}$ ), allows disentangling of the effects of deformation in the entrance channel from the effects of $E_{x}$-dependent shell effects in the dinuclear system. Forming ${ }^{232} \mathrm{Cm}$ in collisions of Ti with deformed $\mathrm{W}$ isotopes at the same $E / V_{B}$ and different $E_{x}$ (and different $E / V_{B}$ for the same $E_{x}$ ) allowed the effects of $E / V_{B}$ and $E_{x}$ to be isolated. Deformation alignment of the $\mathrm{W}$ nuclei plays the major role in determining the observed changes in quasifission characteristics with beam energy (confirming the interpretation of Ref. [10-12]). In the aligned configuration, lower $E_{x}$ appears to slightly broaden the mass-distributions. Twocenter shell model calculations suggest that shell effects may contribute to this dependence.

The experimental mass-angle distributions carry much more information on the dynamics than the mass or angular distributions individually. It is desirable that self-consistent models of reaction dynamics be developed to calculate these distributions, and compare them with the detailed and consistent structures seen experimentally.

The authors acknowledge support from an ARC Discovery Grant.

*Current address: B.A.R.C., Mumbai, India.

${ }^{\dagger}$ Current address: Centro de Física Nuclear da Universidade de Lisboa, Lisboa, Portugal.

[1] Yu. Ts. Oganessian et al., Phys. Rev. C 74, 044602 (2006).

[2] B. B. Back, Phys. Rev. C 31, 2104 (1985).

[3] J. Tōke et al., Nucl. Phys. A 440, 327 (1985).

[4] J. A. Maruhn, W. Greiner, and W. Scheid, in Heavy Ion Collisions, edited by R. Bock (North-Holland, Amsterdam, 1980), Vol. 2, p. 398.

[5] A. Diaz-Torres, Phys. Rev. C 69, 021603(R) (2004); Phys. Rev. C 74, 064601 (2006).

[6] R. Vandenbosch and J. R. Huizenga, Nuclear Fission (Academic Press, New York, 1973).

[7] A. Heinz et al., Nucl. Phys. A 713, 3 (2003).

[8] J. R. Leigh et al., Phys. Rev. C 52, 3151 (1995).

[9] M. Dasgupta et al., Annu. Rev. Nucl. Part. Sci. 48, 401 (1998) and ref. therein.

[10] D. J. Hinde et al., Phys. Rev. Lett. 74, 1295 (1995).

[11] D. J. Hinde et al., Phys. Rev. C 53, 1290 (1996).

[12] D. J. Hinde et al., JNRS 3, 31 (2002).

[13] R. Rafiei et al., Phys. Rev. C 77, 024606 (2008).

[14] R. G. Thomas et al., Phys. Rev. C 77, 034610 (2008).

[15] K. Nishio et al., Phys. Rev. C 63, 044610 (2001).

[16] S. Mitsuoka et al., Phys. Rev. C 65, 054608 (2002).

[17] M. G. Itkis et al., Nucl. Phys. A 734, 136 (2004).

[18] R. Bass, Phys. Rev. Lett. 39, 265 (1977).

[19] B. B. Back et al., Phys. Rev. C 53, 1734 (1996).

[20] R. S. Naik et al., Phys. Rev. C 76, 054604 (2007).

[21] W. Q. Shen et al., Phys. Rev. C 36, 115 (1987).

[22] G. N. Knyazheva et al., Phys. Rev. C 75, 064602 (2007).

[23] A. Diaz-Torres et al., Nucl. Phys. A 679, 410 (2001).

[24] J. Maruhn and W. Greiner, Z. Phys. A 251, 431 (1972).

[25] P. Möller et al., At. Data Nucl. Data Tables 59, 185 (1995).

[26] Ş. Mişicu and W. Greiner, Phys. Rev. C 66, 044606 (2002). 